\title{
Analysis of Suspended Vibrated Drum, Part II: Improvement Dynamic Model Using Particle Swarm Optimization
}

\author{
Dhurgham Ahmed Kadhim ${ }^{1}$ Hussain Abdulaziz Abrahem ${ }^{2}$ and Nazhat Saeed Abdul razaq ${ }^{3}$ \\ Research Scholar ${ }^{1}$ and Assistant Professor $(\mathrm{PhD})^{23}$ \\ Department of Electromechanical Engineering \\ Electromechanical system Engineering \\ University Of Technology \\ Baghdad \\ Iraq
}

\begin{abstract}
Particle swarm optimization (PSO) algorithm has been used to obtain the optimal design of three degrees of freedom mathematical model for the suspended vibrated drum. Optimization is done for the geometric distance of the model; therefore the mathematical model has two constraints which are presented. The constraints must be achieved to improve the model. PSO code is programmed in MATLAB with exchanging data between MATLAB and Simulink.
\end{abstract}

Key Words: PSO, Dynamic model, Optimization, GA.

\section{INTRODUCTION}

Artificial Intelligence (AI) is biologically inspired that simulates natural intelligence of human and animals. Artificial intelligence is a branch of computer science. Any device envisages the environment and takes decision to achieve the goals that called intelligence.

Boyraz and Gunduz (2013) improved their design proposing using genetic algorithm to estimate stiffness, damping coefficient, and geometric distances of suspension elements [1]. Kolhar and Patel (2013) used Solidwork program to optimize the model of suspended drum [2]. Hassaan (2015) used MATLAB optimization toolbox to find optimal parameters of mass drum, spring stiffness and damping coefficient for one degree of freedom (DOF) [3]. Jung and Simpson (2016) used genetic algorithm to multidisciplinary analysis of vibrated drum and washing goals [4].

However according to Couceiro and Ghamisi, Particle swarm optimization is better than genetic algorithm, because genetic algorithm hasn't previous experience and communication of population and it is possible to find local best solution as optimal solution. In addition, if a chromosome is not chosen, the information included by that individual is wasted [5]. Figure (1) shows global and local minimum solutions. PSO is used to improve the 3DOF mathematical model that was presented in reference [6]. The model is shown in figure (2). 


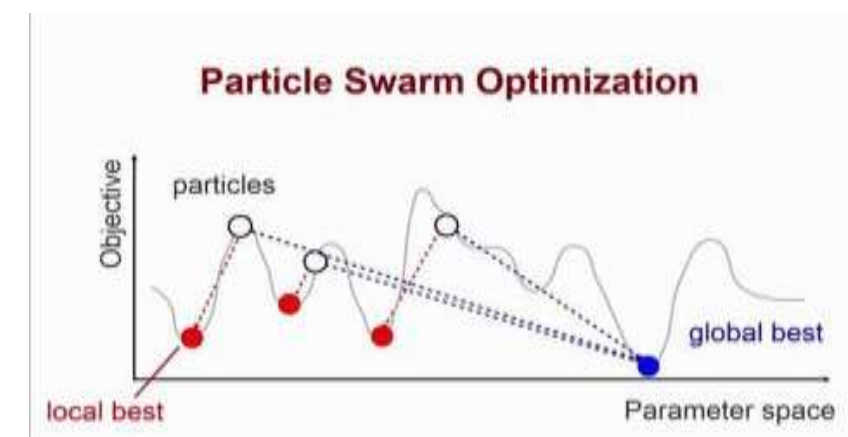

Figure 1: The global and local solution

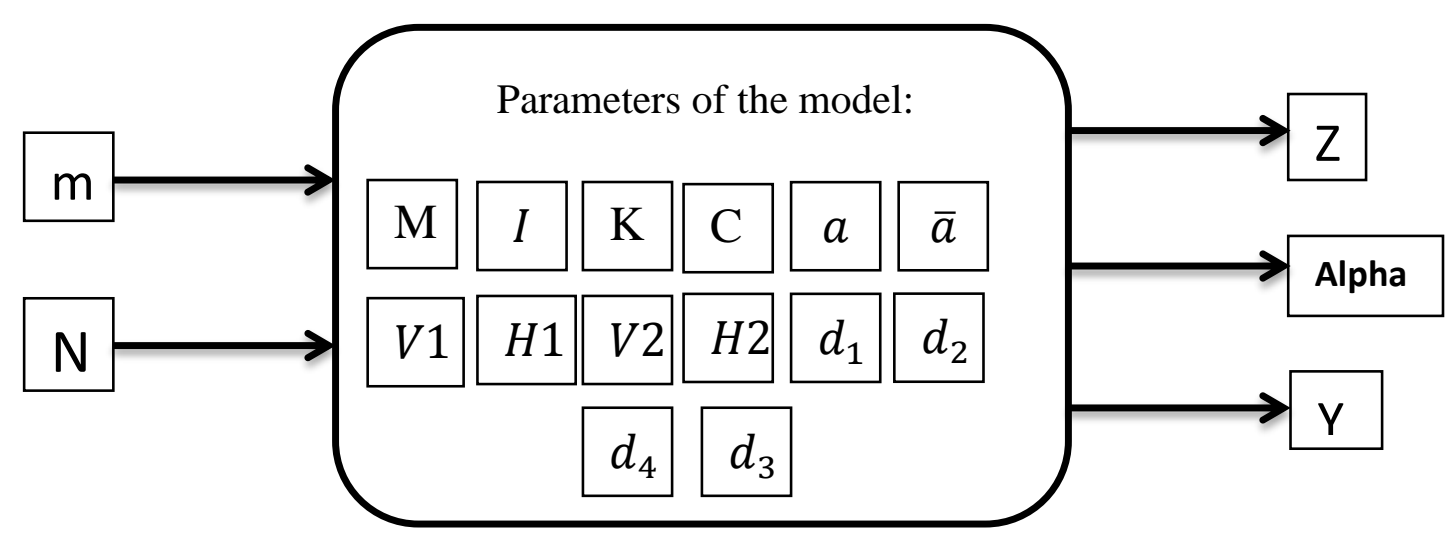

Figure 2: Dynamic model of vibration system

\section{IMPROVEMENT DESIGN USING PSO}

Swarm intelligence simulates searching behavior of the animals group to find optimal source of the food. Particle Swarm Optimization algorithm was discovered by framing social paradigm simulation in simple equations. It is associated to the fishing schooling and bird flocking $[7,8]$. The key point of PSO effective and powerful associated with the previous experience of each particle and communication between populations. Two equations contain a position (solution) and direction (velocity) for each particle in all iterations. The previous experience of a particle is achieved by cognitive component. On the other hand, the communication between populations is done by social component as following:

At iteration $t+1$, the swarm can be updated by [5]:

$v_{i}(t+1)=w * v_{i}(t)+\check{c} r_{1}\left[x_{i}^{*}-x_{i}(t)\right]+\check{c} r_{2}\left[x^{g}-x_{i}(t)\right]$

$x_{i}(t+1)=x_{i}(t)+v_{i}(t+1)$

$i=1, \ldots \ldots, N_{P}$

Where

$x_{i}(t)$ : The position or solution.

$x_{i}(t+1):$ The next position or solution.

$v_{i}(t):$ The velocity.

$v_{i}(t+1):$ The next velocity. 
$N_{P}:$ Number of particles.

$x^{g}$ : The global best solution(gbest).

$x_{i}^{*}$ : Particle best solution(pbest).

$w$ : Inertia coefficient $w=0.99$.

$\check{c}$ : Acceleration (or learning) constant $\check{c}=2, \check{c}>0$.

$r_{1}$ And $r_{2}$ are uniform random numbers within $[0,1]$.

$\check{c} r_{1}\left[x_{i}^{*}-x_{i}(t)\right]:$ Cognitive component.

$\check{c} r_{2}\left[x^{g}-x_{i}(t)\right]$ : Social component.

In every iteration, each particle is updated by following the two best values, namely, the particle best pbest, denoted $x_{i}^{*}, i=$ $1, \ldots, N_{P}$, which is the best solution it has achieved so far, and the global best gbest, denoted $x_{g}$, which is the best value obtained so far by any particle in the population. The best value for the population in a generation is a local best, lbest [5, 7, 8]. The global solution represents the optimal source of the food for birds. The local solution represents any source of food for birds. Constraint represents an enemy for birds that must be avoided to find optimal source of the food [5].

For a model proposing in reference [6], optimization of the Simulink model can be done for each distance $\left(H 1, V 1, d_{1}, d_{2}, H 2, V 2, d_{3}, d_{4}\right)$. Geometric distances are shown in figure (3). The distances $\left(H 1, V 1, d_{1}, d_{2}\right)$ with radius of drum (a) must form a triangle for symmetrical two sides in top half of figure (3). Similarly the distance $\left(H 2, V 2, d_{3}, d_{4}\right)$ with radius of drum (a) must form a triangle for symmetrical two sides in bottom half of figure (3). These conditions are constraints for optimization because probably PSO solution can’t form triangles.

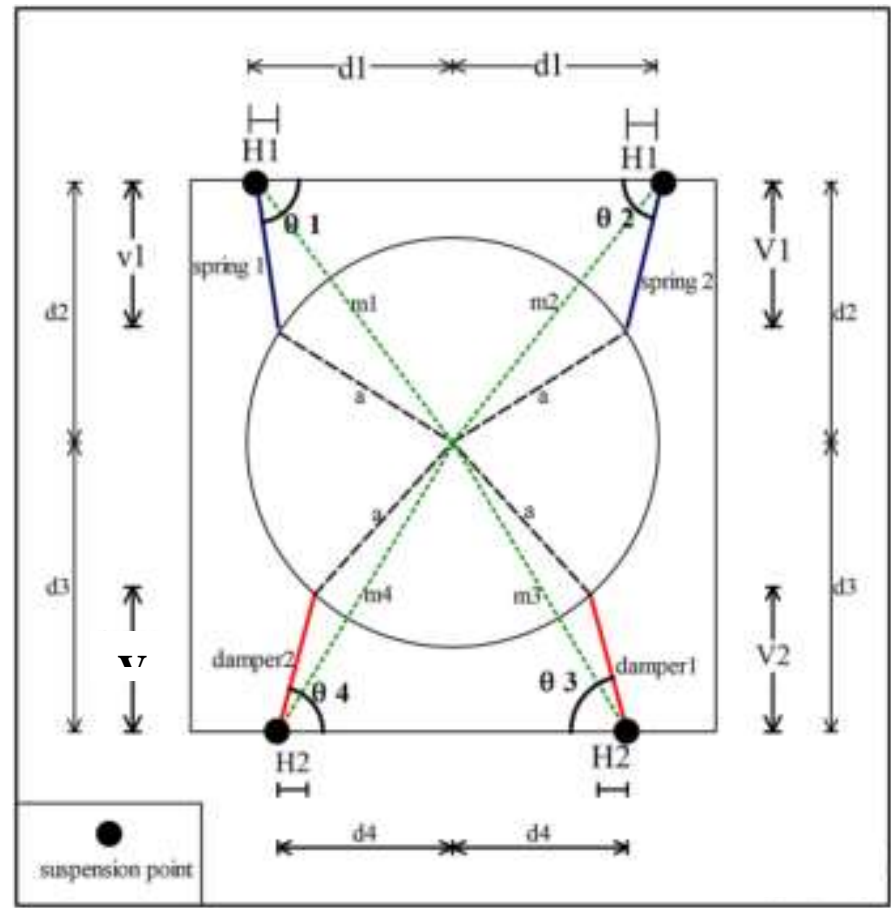

Figure 3: Distances of system

To achieve the triangle in figure (4), geometric distances $\left(H 1, V 1, d_{1}, d_{2}\right)$ process to become $\left(H 1, V 1, m_{1}\right)$ as following:

$L_{1}=\sqrt{(H 1)^{2}+(V 1)^{2}}$

$S=\cos ^{-1} \frac{m_{1}^{2}+L_{1}^{2}-a^{2}}{2 * m_{1} * L_{1}}$

Angle $(s)$ of triangle shown in figure (4): 
To find $d_{1}$ and $d_{2}$ for feeding Simulink model:

$m_{1}=\sqrt{\left(d_{1}\right)^{2}+\left(d_{2}\right)^{2}}$

thus $d_{1}=\sqrt{\left(m_{1}\right)^{2}-\left(d_{2}\right)^{2}}$

$S=\tan ^{-1}\left(\frac{V 1}{H 1}\right)-\tan ^{-1}\left(\frac{d_{2}}{d_{1}}\right)$

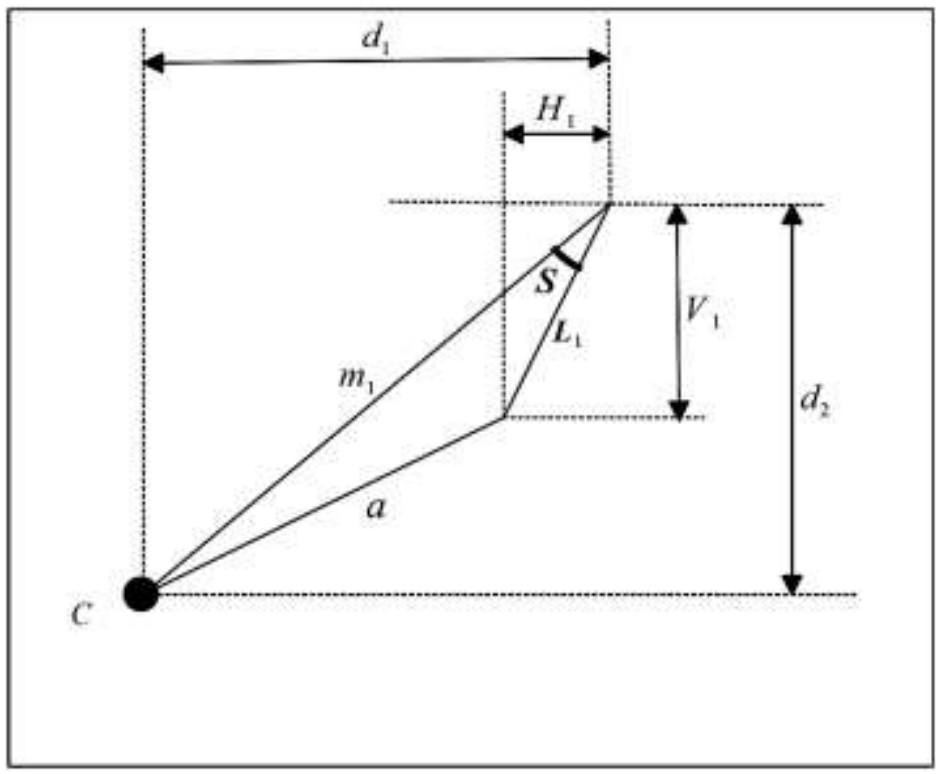

Figure 4: Three triangle side $L_{1}, m_{1}, a$

$S, V_{1}, H_{1}$ are known. And to find $d_{2}$

Solve equation (7) and (8) together to get $d_{2}$ :

Move $\left[\tan ^{-1}\left(\frac{V 1}{H 1}\right)\right]$ term to the left side of equation (8):

$S-\tan ^{-1}\left(\frac{V 1}{H 1}\right)=-\tan ^{-1}\left(\frac{d_{2}}{d_{1}}\right)$

Take (tan function) for each side of above equation

$\tan \left[S-\tan ^{-1}\left(\frac{V 1}{H 1}\right)\right]=-\frac{d_{2}}{d_{1}}$

Thus

$d_{2}=-d_{1} * \tan \left[S-\tan ^{-1}\left(\frac{V 1}{H 1}\right)\right]$

Substitute equation (7) in equation (11):

Thus

$$
d_{2}=\sqrt{\left(m_{1}\right)^{2}-\left(d_{2}\right)^{2}} * \tan \left[\tan ^{-1}\left(\frac{V 1}{H 1}\right)-S\right]
$$

Then

$d_{2}=\frac{m_{1} * \tan \left[\tan ^{-1}\left(\frac{V 1}{H 1}\right)-s\right]}{\sqrt{1+\left(\tan \left[\tan ^{-1}\left(\frac{V 1}{H 1}\right)-s\right]\right)^{2}}}$

In order to solve the constraints problem, the geometric distances shown in figure (6) $\left(H 1, V 1, d_{1}, d_{2}, H 2, V 2, d_{3}, d_{4}\right)$ must be redefined to become as $\left(H 1, V 1, m_{1}, H 2, V 2, m_{3}\right)$ that shown in figure (7) with notification that $m_{1}=m_{2}, m_{3}=m_{4}$. 


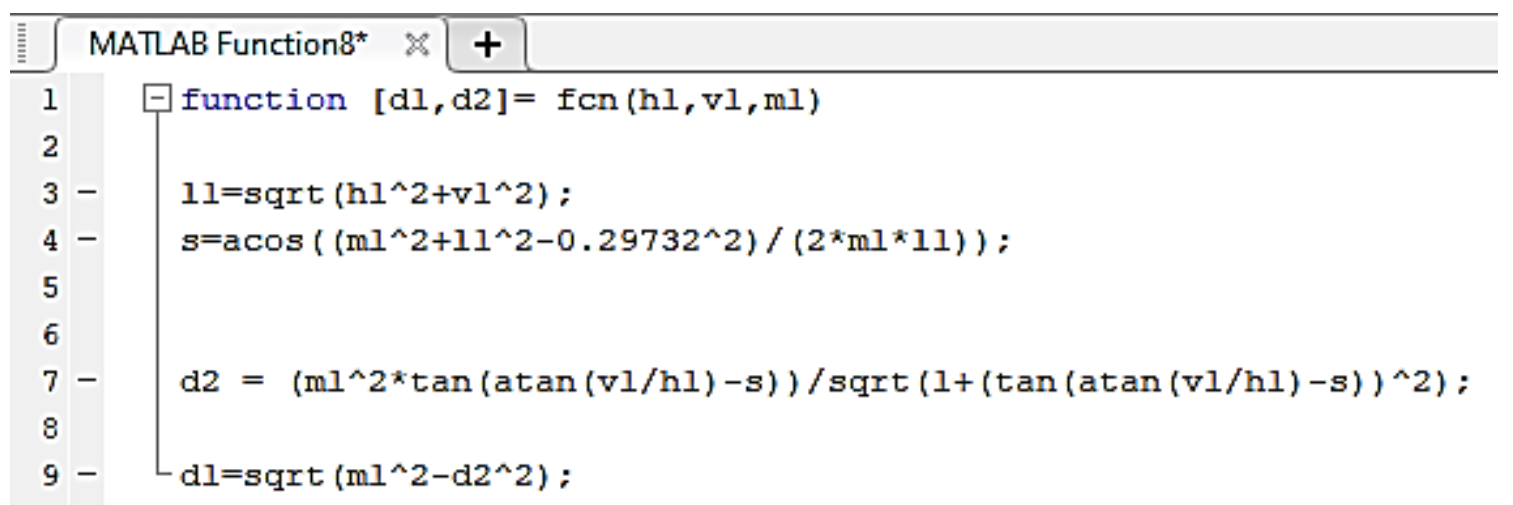

Figure 5: Equations (3) (4) (7) and (13) programed in MATLAB function block

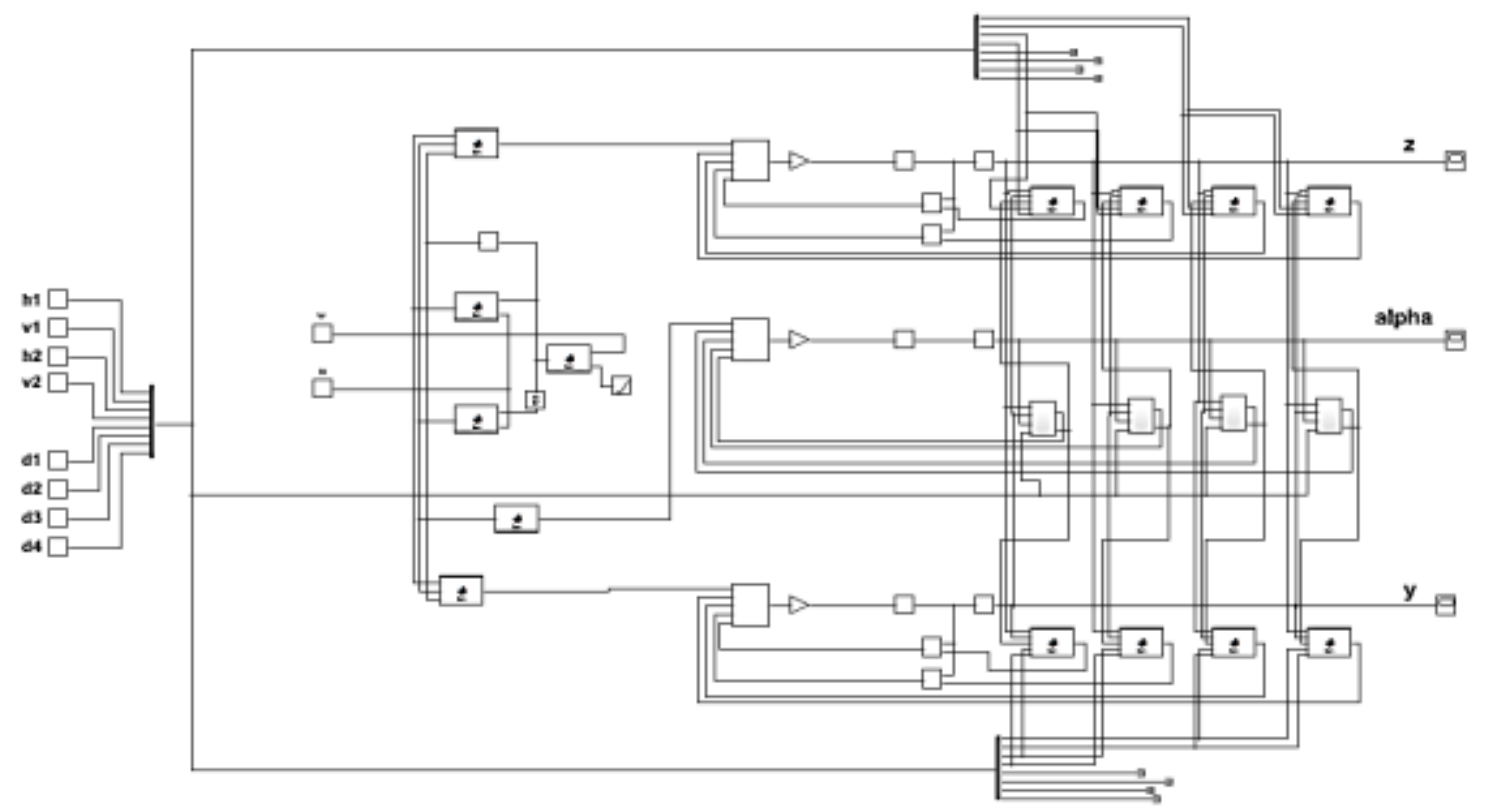

Figure 6: Simulink model of 3DOF vibration system with the geometric distances as inputs

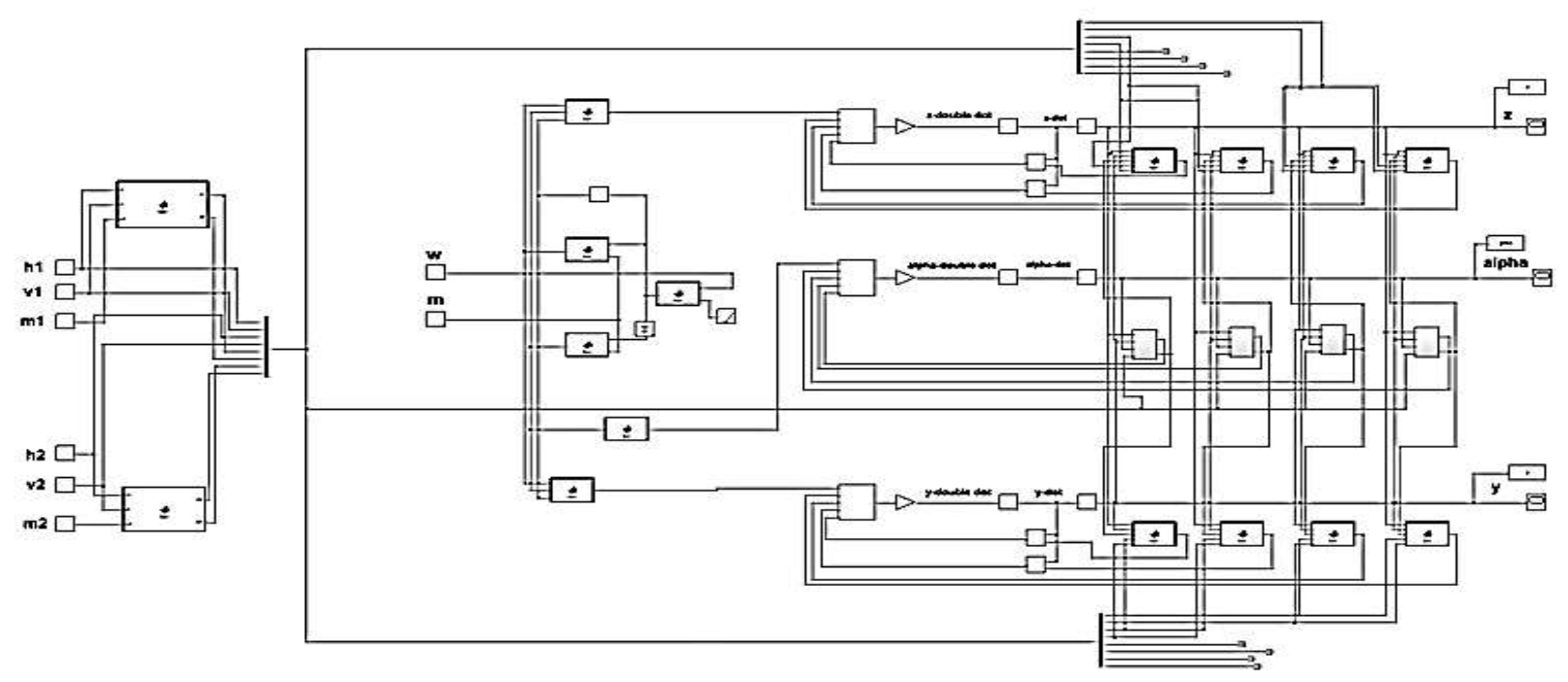

Figure 7: Inputs $\left(H 1, V 1, m_{1}, H 2, V 2, m_{2}\right)$ for Simulink model

An Optimization problem is a code of PSO in MATLAB but the model in Simulink. To solve this problem, workspace of the MATLAB must be shared with the workspace of Simulink then Simulink was run by sim command with sending the output of 
Simulink to workspace by (simout block) for each degree of freedom (z, y, and alpha). Each degree of freedom (z, y, and alpha) represent as a matrix in MATLAB, then max value in transient and steady state is found to comparison. To optimize the 3DOF Simulink model, Boyraz and Gündüz [1] proposed three type of criterions for minimizing amplitude of the Simulink output, which are:

- Transient vibration.

- Wobbling of drum movements.

- Transient with steady-state vibration.

The best criterion is using the steady-state and transient amplitudes of the vibration displacements. The cost (or fitness) function according to the best criterion is:

output $=$ z.output + alpha.output + y.output

Where

z. output $=$ z.transient + z. steady state

alpha. output $=$ alpha.transient + alpha. steady state

y.output $=y$. transient $+y$. steady state

Equation (14) used in $[1,4]$ but without alpha term. PSO algorithm steps are shown in figure (8).

1. Set $t=1$.

Initialize each particle in the population by randomly selecting values for its position $\boldsymbol{x}_{i}$ and velocity $\boldsymbol{v}_{i}, i=1, \ldots, N_{P}$.

2. Repeat:

a. Calculate the fitness value of each particle $i$.

If the fitness value for each particle $i$ is greater than its best fitness value found so far, then revise $\boldsymbol{x}_{i}^{*}(t)$.

b. Determine the location of the particle with the highest fitness and revise $x^{g}(t)$ if necessary.

c. for each particle $i$, calculate its velocity according to equation (1)

d. Update the location of each particle $i$ according to equation (2)

e. Set $t=t+1$.

until stopping criteria are met.

Figure 8: PSO stens

\section{NATURAL FREQUENCIES}

To find natural frequencies of any system which is modeled in MATLAB or Simulink, the command damp is used which is shown in figure (9) after system linearization that shows in figure (2) to become as state space model.

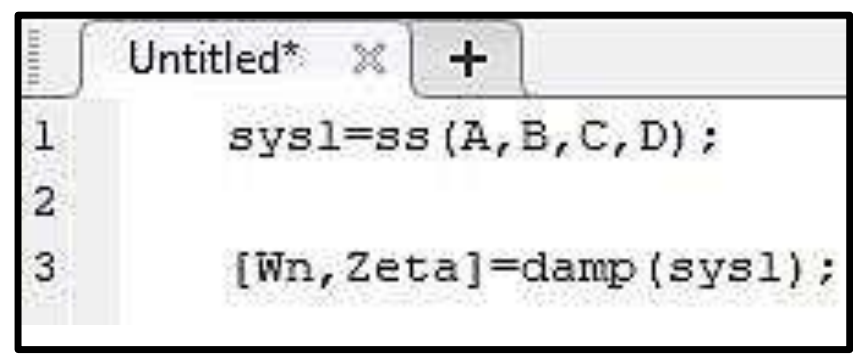

Figure 9: MATLAB command using to find natural 


\section{RESULTS}

Figure (10) shows the relationship between cost function and iteration. It should be noted that the cost function changes with the variation of geometric distance. Figure (11) shows results of Natural frequencies ( $\mathrm{rad} / \mathrm{s})$ and damping ratios form state space model. Figures (12), (13) and (14) show comparison of Simulink outputs between first simulation and optimization.

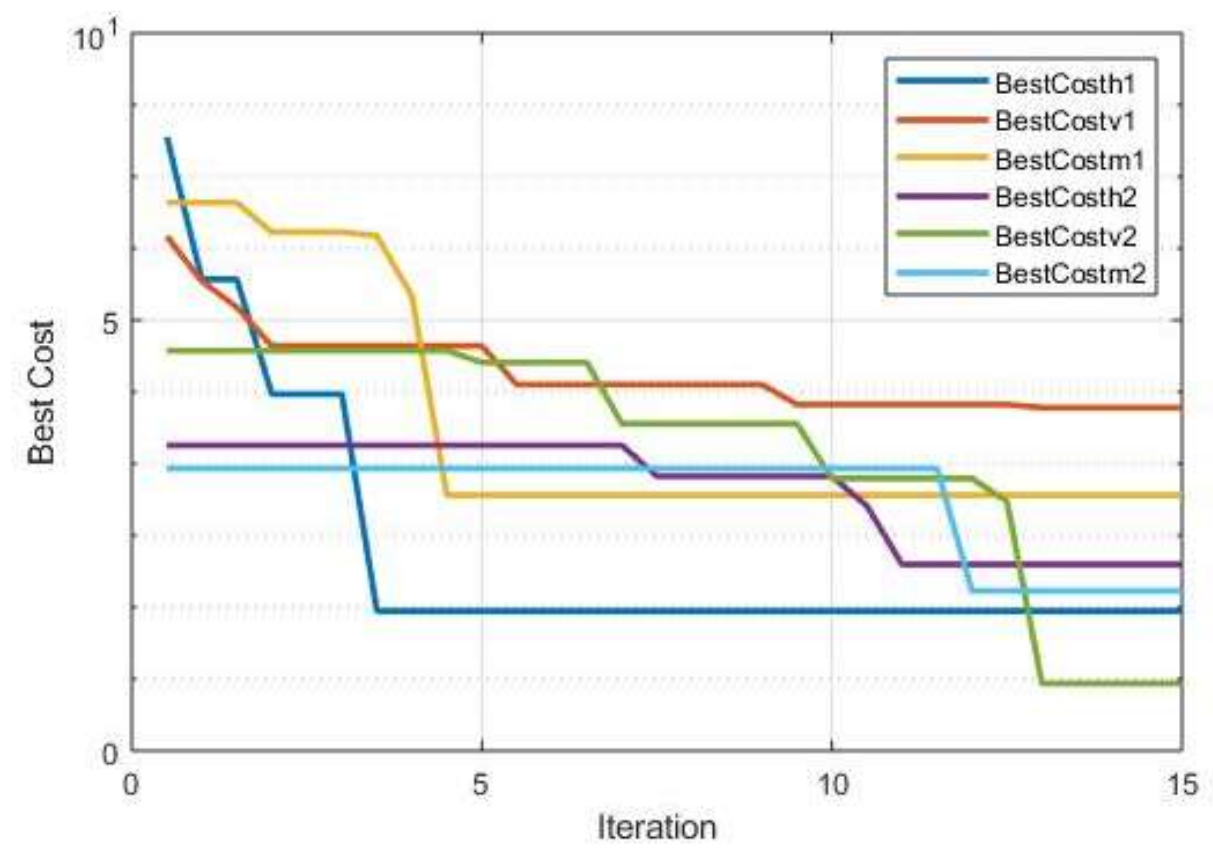

Figure 10: Relationship between cost function and iterations

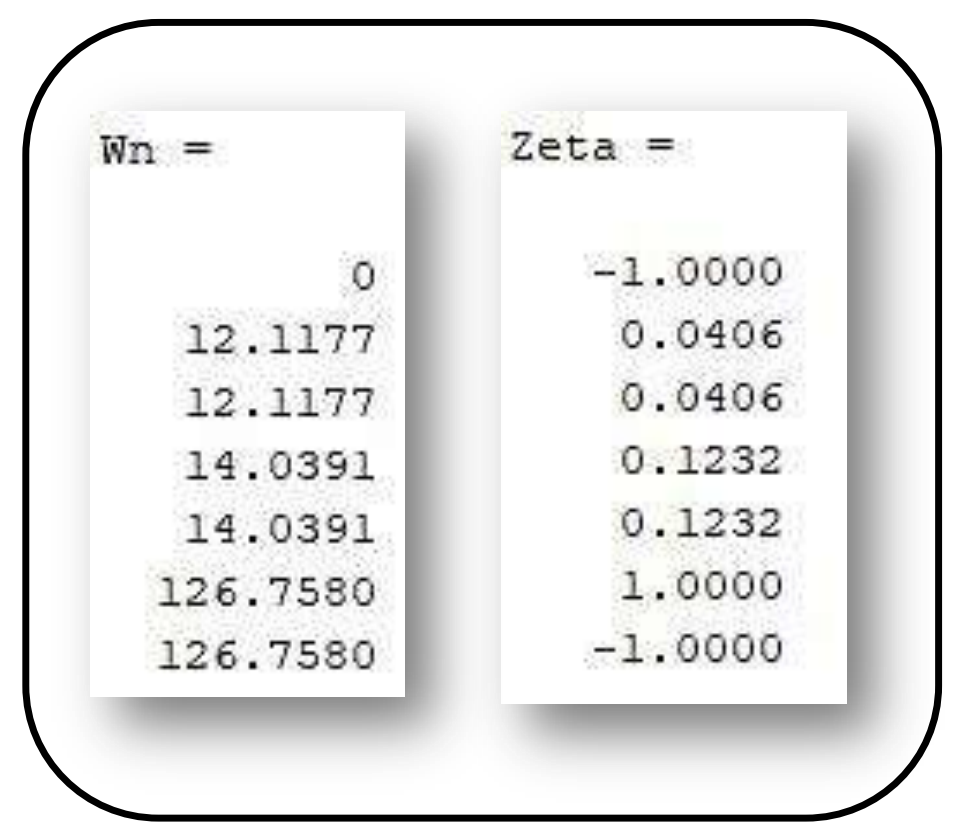

Figure 11: Results of Natural frequencies $(\mathrm{rad} / \mathrm{s})$ and damping ratios form state space model 
International Journal of Advances in Scientific Research and Engineering (ijasre), Vol 5 (1), January-2019

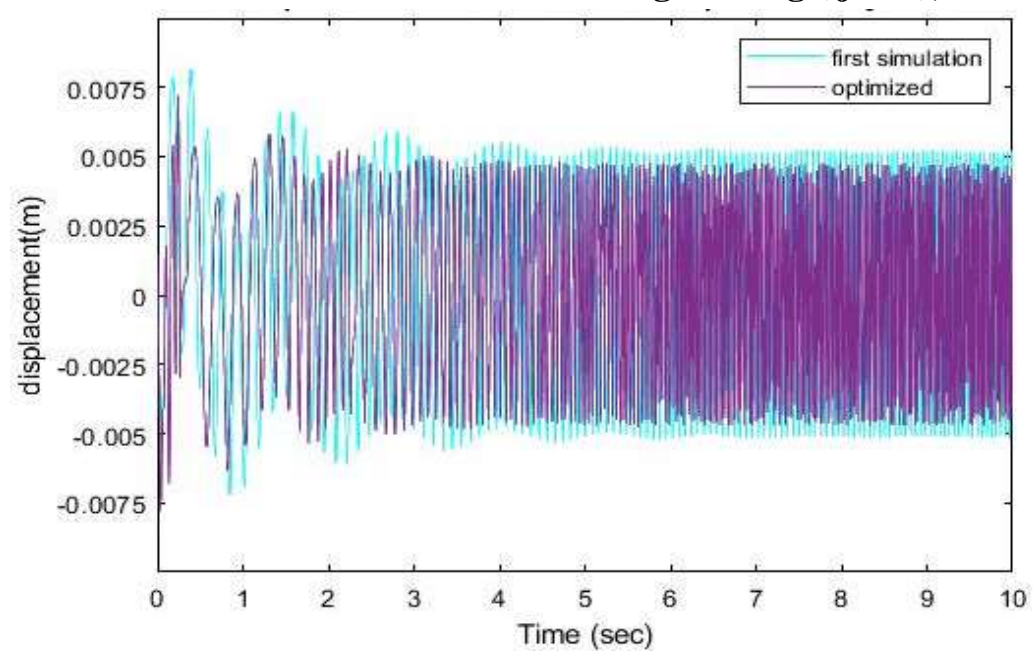

Figure 12: Comparison of y-axis between simulation and optimization

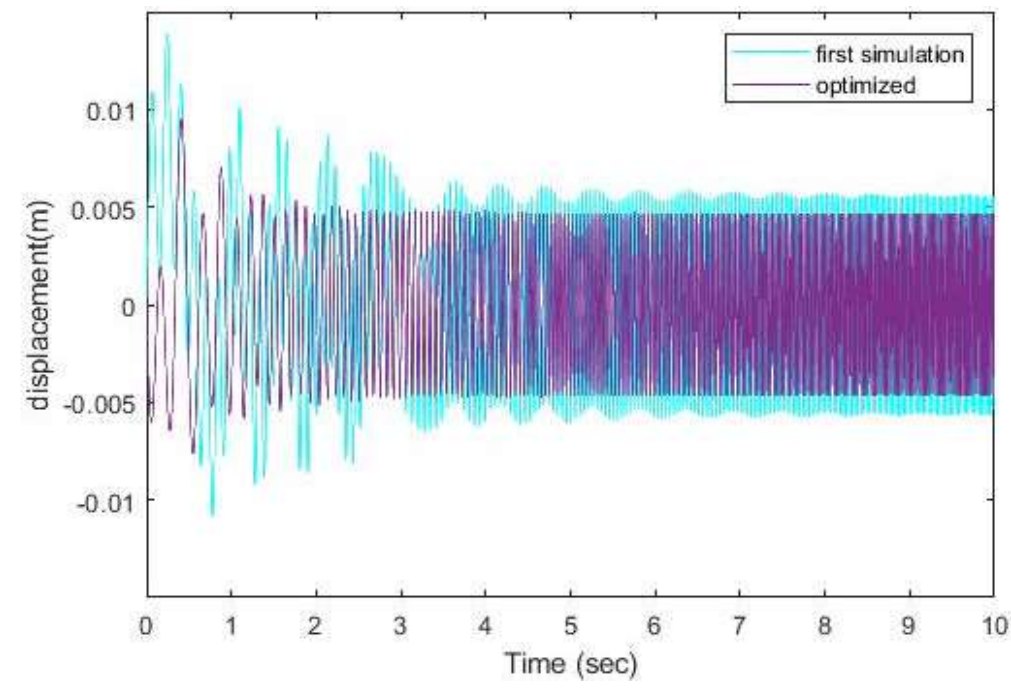

Figure 13: Comparison of z-axis between simulation and optimization

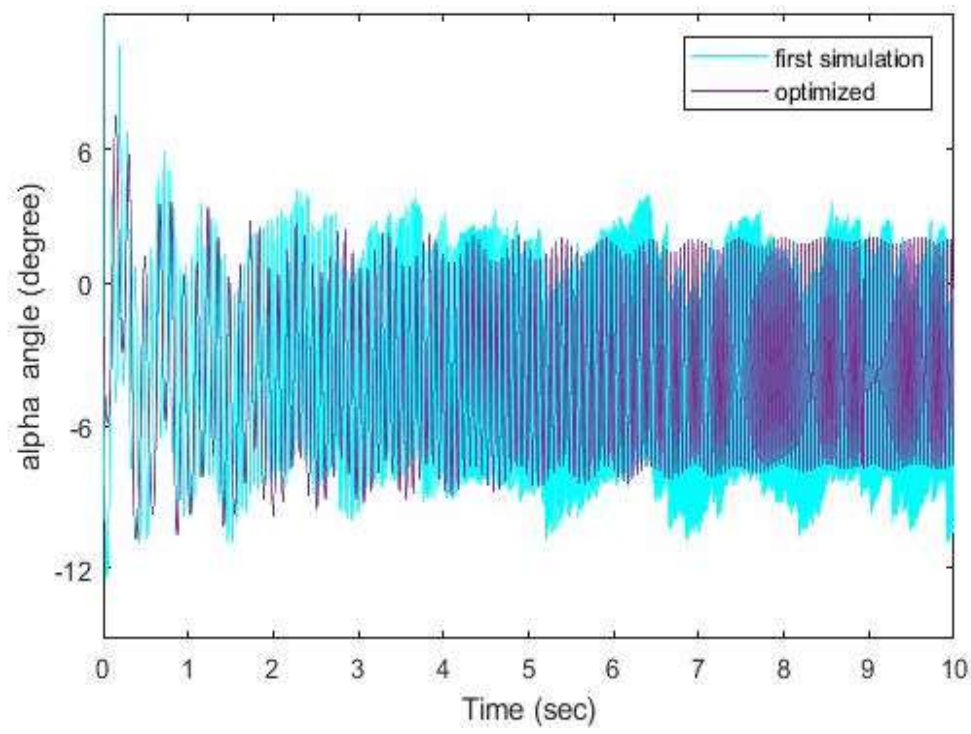

Figure 14: Comparison of alpha angle between simulation and optimization 


\section{DISCUSSION}

- Minimum solutions have been obtained successfully by changing geometric distances as shown in figures (12-13).

- For achieving the model constraints then the geometric distances $\left(H 1, V 1, d_{1}, d_{2}, H 2, V 2, d_{3}, d_{4}\right)$ convert to become $\left(H 1, V 1, m_{1}, H 2, V 2, m_{2}\right)$.

- PSO reaches the optimal solution according to figure (10) that shows the constant values of cost function near to iteration 14.

- Number of natural frequencies must be equal to degrees of freedom of the model but the repetition of natural frequencies values in figure (11)) relates to the number of the state space vectors. Furthermore, the state vectors depend on the number of the integrations and derivations in the Simulink model.

\section{CONCLUSION}

1. Efficient solutions have been obtained successfully by particle swarm optimization.

2. The equations which are derived are successful for solving the constraints of the mathematical model.

3. PSO reaches the optimal solution according to the relationship between cost function and iterations which shows the constant values of each cost function near to iteration 14.

\section{REFERENCES}

[1] Boyraz P, Gündüz M. Dynamic modeling of a horizontal washing machine and optimization of vibration characteristics using Genetic Algorithms. Mechatronics. 2013 Sep 1;23(6):581-93.

[2] Kolhar SS, Patel DR. Optimization of a drum type washing machine by analytical and computational assessment. International Journal of Scientific and Engineering Research. 2013 Jun;4(6):2759-63.

[3] Hassaan GA. Vibration Analysis of a Horizontal Washing Machine, Part III: Optimal Parameters. International Journal of Engineering and Techniques. 2015 Oct 1(5).

[4] Jung S, Simpson TW. Multidisciplinary analysis and product family optimization of front-loading washing machines. InASME 2016 International Design Engineering Technical Conferences and Computers and Information in Engineering Conference 2016 Aug 21 (pp. V02BT03A039-V02BT03A039). American Society of Mechanical Engineers.

[5] Couceiro M, Ghamisi P. Fractional order darwinian particle swarm optimization: applications and evaluation of an evolutionary algorithm. Springer; 2015 Jun 16.

[6] Kadhim DA, Abrahem HA, Abdul razaq NS. Analysis of Suspended Vibrated Drum, Part I: Modelling with Full Plane for Drum Vibration. International Journal of Engineering and Techniques. 2019, Jan-Feb 5(5), pp.13-24.

[7] Du KL, Swamy MN. Search and optimization by metaheuristics. Birkhaüser) July. 2016.

[8] Eberhart R, Kennedy J. A new optimizer using particle swarm theory. InMicro Machine and Human Science, 1995. MHS'95., Proceedings of the Sixth International Symposium on 1995 Oct 4 (pp. 39-43). IEEE. 